\title{
AVALIAÇÃO DO AQUECIMENTO ELÉTRICO RESISTIVO APLICADO A UM RESERVATÓRIO DE ÓLEO PESADO
}

\author{
A. A. R. DINIZ ${ }^{1}$, E. A. ARAÚJO ${ }^{1}$ e J. L. M. BARILLAS ${ }^{1}$ \\ ${ }^{1}$ Universidade Federal do Rio Grande do Norte, Programa de Pós-Graduação em Ciência e \\ Engenharia de Petróleo \\ E-mail para contato: anthony_andrey@yahoo.com
}

\begin{abstract}
RESUMO - Na indústria do petróleo, o óleo pesado possui produção mais difícil e onerosa. Dentre os processos de recuperação avançada para extração desse tipo de óleo, destaca-se a injeção de vapor, que embora lidere em desenvolvimento e aplicação, possui algumas restrições quanto à aplicação.O uso da energia elétrica também é de interesse, por oferecer menores restrições com respeito aos candidatos para sua aplicação, quando comparada aos métodos convencionais de injeção de vapor. Nesse trabalho foi estudado um modelo de reservatório semissintético, homogêneo, sem presença de aquífero, saturado com óleo de $16,2^{\circ}$ API. Através de um planejamento fatorial completo, do tipo $3^{4}$, investigou-se a influência de dois parâmetros de reservatório e dois parâmetros operacionais no fator de recuperação de óleo. Ao final, em termos de parâmetros de reservatório, concluiu-se que maior nível de saturação de água conata, com maior teor de salinidade, favorece a produção de óleo. Em termos operacionais, a adoção de uma configuração trifásica, com maior nível de tensão, maximiza a produção de óleo. Entretanto, sugere-se a complementação desse estudo por uma avaliação econômica, para verificar se a melhor configuração operacional favorece o maior retorno financeiro.
\end{abstract}

\section{INTRODUÇÃO}

$\mathrm{Na}$ indústria do petróleo, o óleo pesado tem sido geralmente um recurso preterido, devido à sua produção ser mais difícil e onerosa. Entretanto, essas reservas merecem uma atenção especial, pois são atribuídos mais de 6 trilhões de barris de óleo "in place" aos hidrocarbonetos mais pesados, ou seja, o triplo da quantidade combinada das reservas mundiais convencionais de óleo e gás (CURTIS et al., 2002).

A alta viscosidade é uma grande preocupação para a recuperação de reservatórios de óleo pesado. Um óleo de viscosidade muito alta torna um projeto tecnicamente desafiador e provoca impacto no seu retorno financeiro (CARRIZALES, 2010). As vastas quantidades de óleo pesado e ultrapesado dominam as reservas mundiais de hidrocarbonetos, mas o óleo e o gás convencionais, por serem mais facilmente produzidos, ultrapassam essa contraparte de menor energia, em termos dos níveis atuais de produção. Muitas reservas dos hidrocarbonetos mais pesados estão à espera de novas tecnologias que os tornem em projetos economicamente viáveis (CURTIS et al., 2002).

Vários processos de recuperação avançada (EOR) são utilizados para produzir óleo pesado, sendo esses métodos normalmente divididos em duas categorias: químicos e térmicos. Os processos químicos incluem a injeção de gás miscível, injeção de alcalinos ou de polímeros, que 
reagem quimicamente com o óleo tanto para reduzir a tensão interfacial no reservatório, como no caso da injeção de alcalinos, ou aumentar o varrido vertical, no caso da injeção de polímeros (PERASER et al., 2012). Os métodos térmicos constituem a principal técnica em uso para recuperar óleos pesados e viscosos, uma vez que o aumento de temperatura reduz drasticamente a viscosidade do óleo, afinando-o e melhorando sua fluidez e mobilidade no interior do reservatório (KOVSCEK, 2012).

Os métodos térmicos envolvem processos bem conhecidos, como é o caso da injeção de vapor (cíclica, contínua, SAGD e ES-SAGD), combustão in situ, e uma técnica mais recente, que consiste em aquecer o reservatório com energia elétrica (CARRIZALES, 2010).

Embora a injeção de vapor lidere em desenvolvimento e aplicação, a aplicação de energia elétrica é de interesse, por oferecer menores restrições com respeito aos candidatos para sua aplicação, quando comparada aos métodos convencionais de injeção de vapor (estimulação cíclica e contínua) (CARRIZALES, 2010). No método térmico utilizando aquecimento eletromagnético, transforma-se energia elétrica em energia térmica, aumentando a temperatura média do reservatório, reduzindo a viscosidade dos fluidos e, consequentemente, aumentando a mobilidade da fase óleo (SANTOS, 2010).

Nesse contexto, esse trabalho tem como objetivo o estudo da aplicação do aquecimento resistivo em um reservatório de óleo pesado, inclusive a avaliação da influência de dois parâmetros de incerteza do reservatório (saturação de água conata e salinidade da água) e dois parâmetros operacionais (nível de tensão e configuração elétrica), na recuperação do óleo existente no reservatório.

\section{MATERIAIS E MÉTODOS}

Para este estudo foi modelado um reservatório homogêneo, semissintético, com dimensões de $200 \times 200 \times 20 \mathrm{~m}^{3}, 25 \%$ de porosidade, permeabilidade horizontal de $1.000 \mathrm{mD}$, e permeabilidade vertical de $10 \%$ da horizontal, sem a presença de aquífero. O fluido utilizado foi um óleo com densidade de $16,2^{\circ}$ API, modelado com 2 componentes e 6 pseudocomponentes. As modelagens do fluido e do reservatório foram realizadas utilizando os módulos WINPROP e BUILDER, respectivamente, com simulações realizadas no STARS, sendo todos da CMG Suite v.2012

O reservatório é mostrado na Figura 1a, onde foi perfurado um arranjo "five-spot" de poços, com um eletrodo em cada um deles, penetrando $10 \mathrm{~m}$ no reservatório. Em um tempo de projeto de 15 anos, foram avaliados os efeitos no fator de recuperação de dois parâmetros de incerteza do reservatório (teor de salinidade da água e saturação de água conata) e dois parâmetros operacionais (configuração de excitação dos eletrodos e nível de tensão aplicado).

As configurações de excitação dos eletrodos foram definidas com base no esquema mostrado na Figura 1b, com as configurações listadas na Tabela 1.

\subsection{Aquecimento Elétrico Resistivo}

Nesse método, uma corrente de baixa frequência, utilizando o mecanismo de condução iônica, é injetada no reservatório para fluir através da água intersticial presente no sistema matriz do reservatório. O calor é gerado através da conversão da energia elétrica em calor, através das 
perdas ôhmicas da formação (SIERRA et al., 2001). A Figura 2 apresenta um diagrama esquemático de um sistema de aquecimento resistivo.

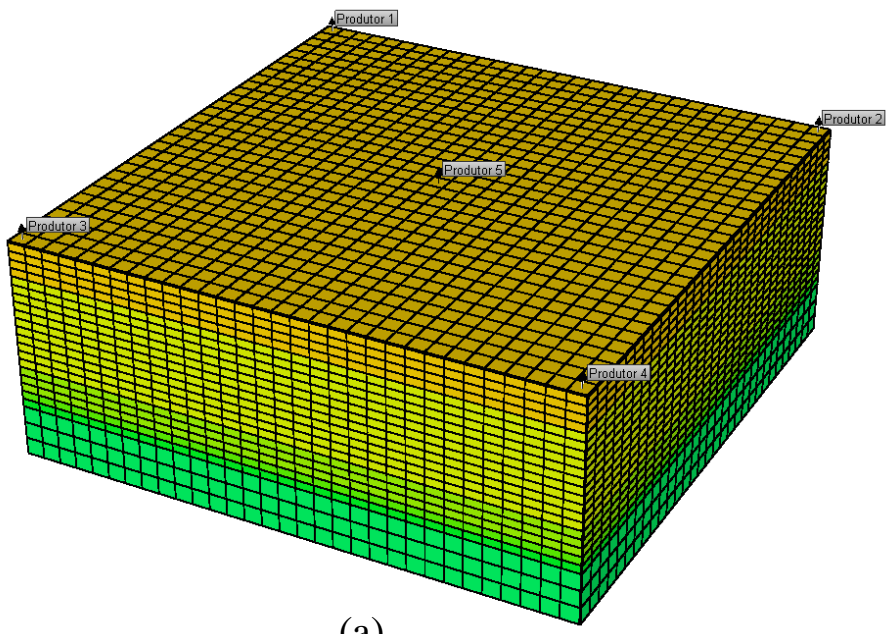

(a)

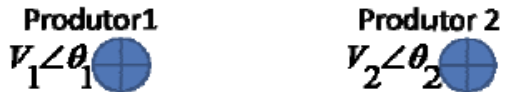

Produtor5 $V_{5}<\theta_{5}$

Produtor3 $V_{3} \angle \theta_{3}$

(b)

Figura 1. (a) Visão geral do modelo de reservatório e (b) configuração para ligação dos eletrodos (OLIVEIRA, 2009).

Tabela 1. Configurações de alimentação elétrica

\begin{tabular}{c|c|c|c|c|c}
\hline Configuração & Produtor 1 & Produtor 2 & Produtor 3 & Produtor 4 & Produtor 5 \\
\hline Geral & $\mathrm{V}_{1} \angle \theta_{1}$ & $\mathrm{~V}_{2} \angle \theta_{2}$ & $\mathrm{~V}_{3} \angle \theta_{3}$ & $\mathrm{~V}_{4} \angle \theta_{4}$ & $\mathrm{~V}_{5} \angle \theta_{5}$ \\
\hline Monofásica & 0 & 0 & 0 & 0 & $\mathrm{~V} \angle 0^{\circ}$ \\
\hline Trifásica & $\mathrm{V} \angle 120^{\circ}$ & $\mathrm{V} \angle 120^{\circ}$ & $\mathrm{V} \angle 240^{\circ}$ & $\mathrm{V} \angle 240^{\circ}$ & $\mathrm{V} \angle 0^{\circ}$ \\
\hline Bifásica & $\mathrm{V} \angle 0^{\circ}$ & $\mathrm{V} \angle 120^{\circ}$ & $\mathrm{V} \angle 120^{\circ}$ & $\mathrm{V} \angle 0^{\circ}$ & $\mathrm{V} \angle 0^{\circ}$ \\
\hline
\end{tabular}

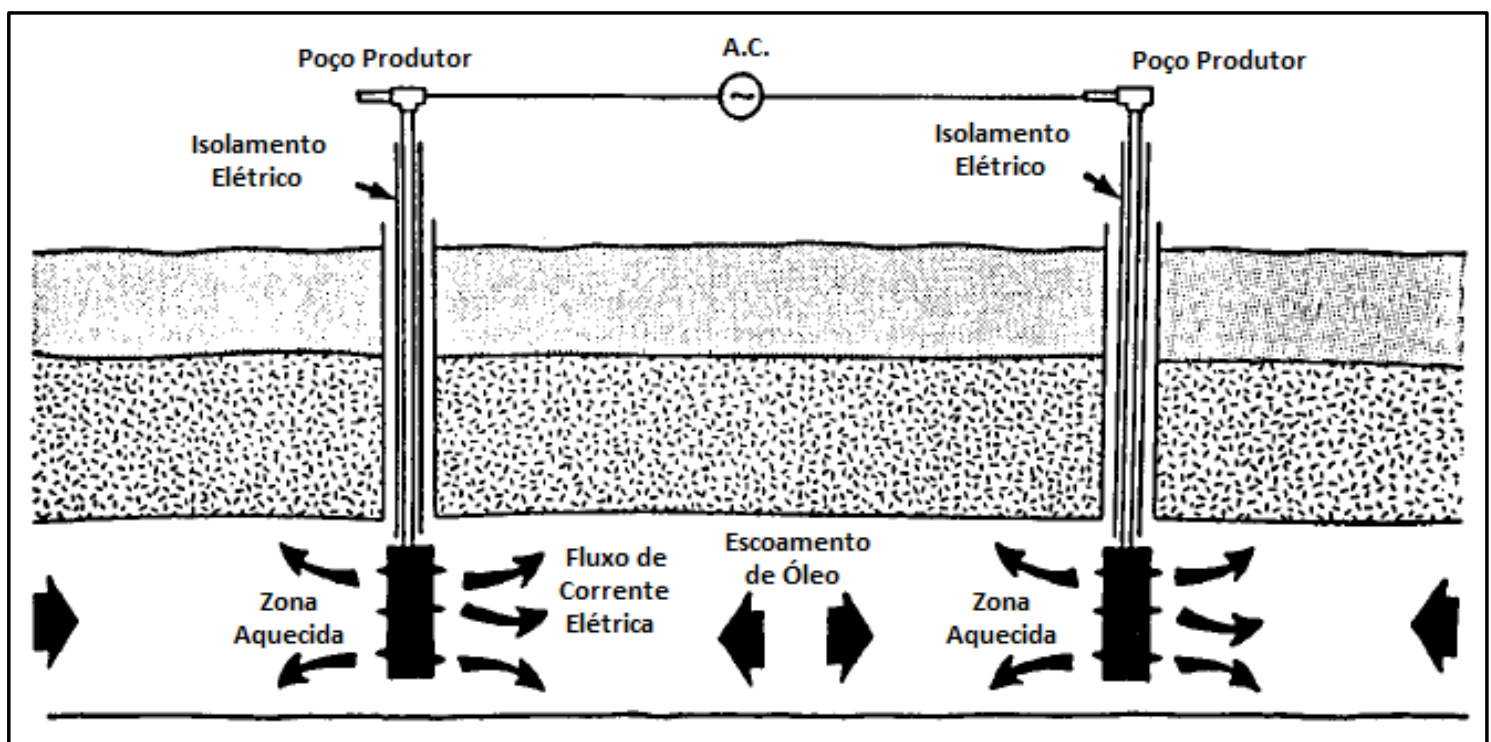

Figura 2. Diagrama esquemático do aquecimento elétrico resistivo.

Fonte: Adaptado de Pizarro e Trevisan (1990).

Baylor, Maggard e Wattenbarger (1990) destacam que, devido ao caminho da corrente ser 
providenciado pela água in situ, as temperaturas na formação devem ser mantidas abaixo do ponto de ebulição da água, para que a continuidade elétrica seja mantida.

\section{RESULTADOS E DISCUSSÕES}

Nas simulações foram considerados três nível de salinidade, tendo sido um deles escolhido com base em informações de salinidade nos reservatório do pré-sal; três níveis de saturação de água conata do reservatório; três configurações de excitação dos eletrodos e três níveis de tensão elétrica. Os parâmetros e níveis avaliados estão listados na Tabela 2.

Tabela 2. Parâmetros e níveis avaliados na análise de sensibilidade

\begin{tabular}{l|c|c|c}
\hline Parâmetros & $\mathbf{- 1}$ & $\mathbf{0}$ & $\mathbf{+ 1}$ \\
\hline Saturação de água conata & $20 \%$ & $30 \%$ & $40 \%$ \\
\hline Salinidade da água & $400 \mathrm{ppm}$ & $30.000 \mathrm{ppm}$ & $250.000 \mathrm{ppm}$ \\
\hline Configuração elétrica & Monofásica & Trifásica & Bifásica \\
\hline Nível de tensão & 55 VAC & 82,5 VAC & $110 \mathrm{C}$ \\
\hline
\end{tabular}

Para a análise de sensibilidade do fator de recuperação foi aplicado um planejamento fatorial do tipo $3^{4}$, onde foram estudados 81 casos. O diagrama de Pareto obtido para os 15 anos de projeto é mostrado na Figura 3. Também foram construídos diagramas para 5 e 10 anos, mas indicaram resultados semelhantes ao obtido no tempo final de projeto, por isso não foram reproduzidos.

A partir do Pareto foram verificadas as interações estatisticamente significativas entre os parâmetros avaliados, de onde se constatou a significância estatística das seguintes interações: nível de tensão e configuração elétrica; nível de tensão e saturação de água conata no reservatório; nível de tensão e teor de salinidade da água; configuração elétrica e teor de salinidade da água; configuração elétrica e saturação de água conata; e a interação entre saturação de água conata e teor de salinidade da água.

Avaliando a superfície de resposta de interação entre o nível de tensão e a configuração elétrica adotada, mostrada na Figura 4a, verifica-se que o maior fator de recuperação $(62,4 \%)$ foi obtido quando se optou mais uma vez pela configuração elétrica do nível intermediário (trifásica), excitada pelo maior nível de tensão (110 volts).

Para a superfície de resposta da interação entre nível de tensão e saturação de água conata do reservatório, mostrada na Figura 4b, observa-se que o maior fator de recuperação $(61,3 \%)$ foi obtido excitando os eletrodos com o maior nível de tensão e com o reservatório contendo maior saturação de água conata (40\%).

Avaliando a superfície de resposta de interação entre o nível de tensão e o teor de salinidade da água, apresentada na Figura 5a,constata-se que o maior fator de recuperação $(61,3 \%)$ foi obtido como resultado da excitação dos eletrodos com o maior nível de tensão, em um ambiente com maior teor de salinidade (250.000 ppm).

Para o caso da superfície de resposta de interação entre a configuração elétrica e o teor de salinidade da água,mostrada na Figura 5b,verifica-se que o maior fator de recuperação $(59,1 \%)$ foi 
obtido devido à adoção da configuração elétrica trifásica (nível intermediário do parâmetro) no reservatório com água de maior teor de salinidade.

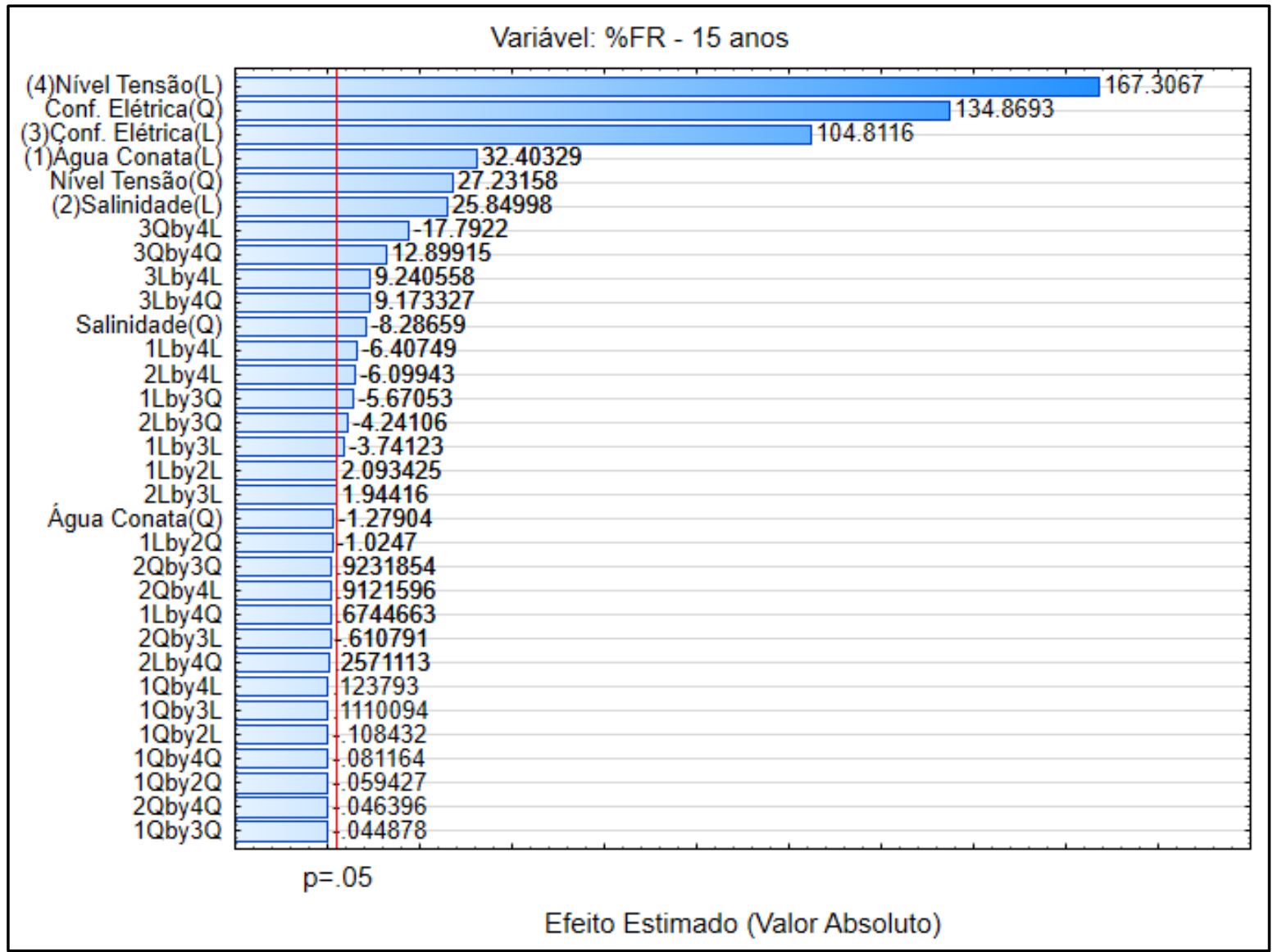

Figura 3. Diagrama de Pareto gerado para a análise do fator de recuperação considerando 15 anos de projeto.

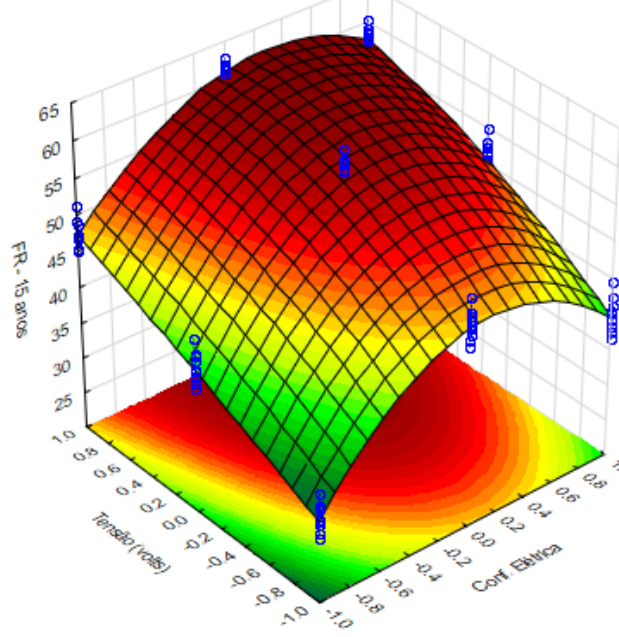

(a)

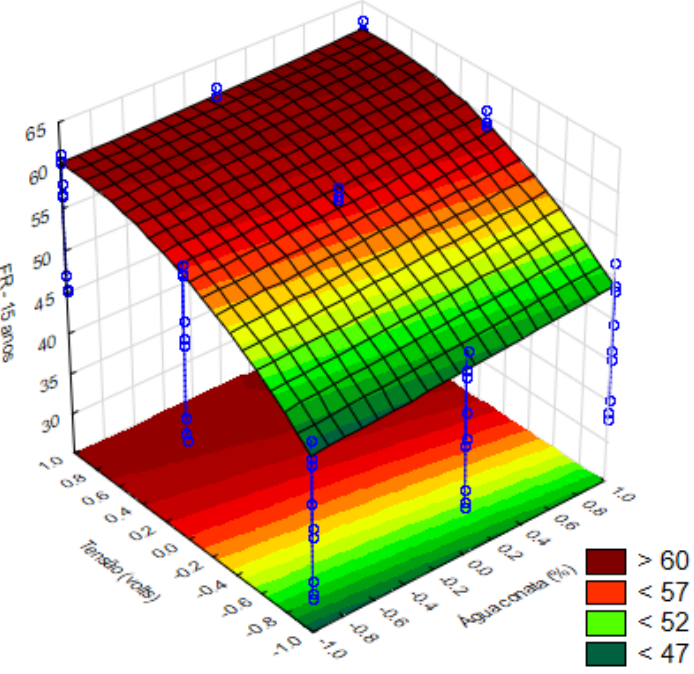

(b)

Figura 4. Superfícies de resposta de interação entre: (a) nível de tensão e configuração elétrica de excitação dos eletrodos e (b) nível de tensão e saturação de água conata do reservatório. 


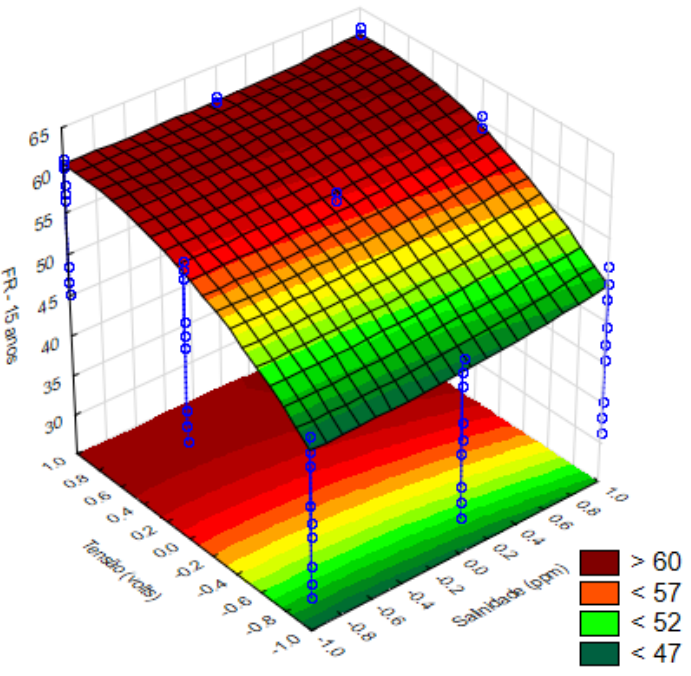

(a)

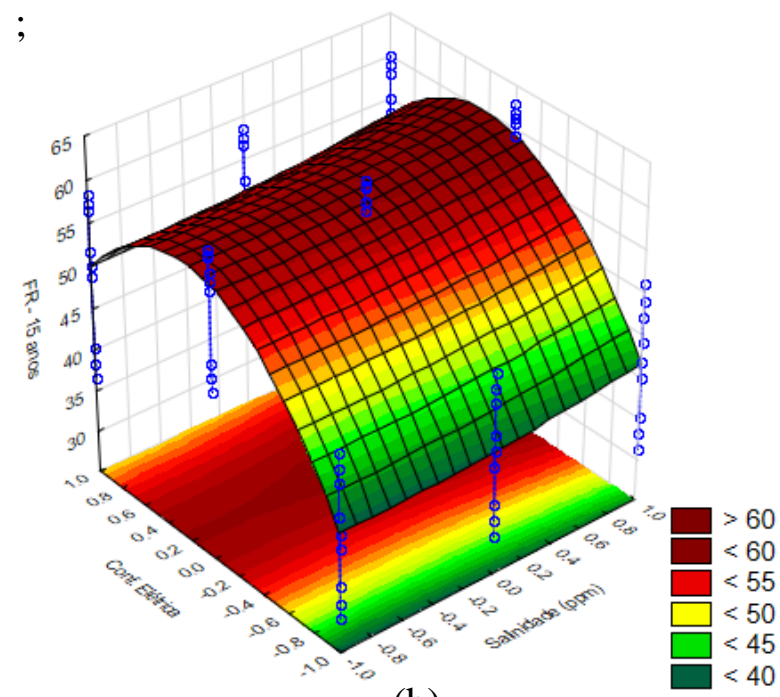

(b)

Figura 5. Superfícies de resposta de interação entre: (a) nível de tensão e teor de salinidade da água e (b) configuração elétrica de excitação dos eletrodos e teor de salinidade da água do reservatório.

Para a superfície de resposta da interação entre configuração elétrica e saturação de água conata do reservatório, mostrada na Figura 6a, o maior fator de recuperação $(58,8 \%)$ foi obtido adotando-se a configuração elétrica de nível intermediário (trifásica), com o reservatório e mantendo a maior saturação de água conata.

Para a superfície de resposta da interação entre o teor de salinidade da água e a saturação de água conata do reservatório, apresentada na Figura $6 \mathrm{~b}$, o maior fator de recuperação $(60,6 \%)$ foi obtido no reservatório com maior saturação de água conata e maior teor de salinidade da água.

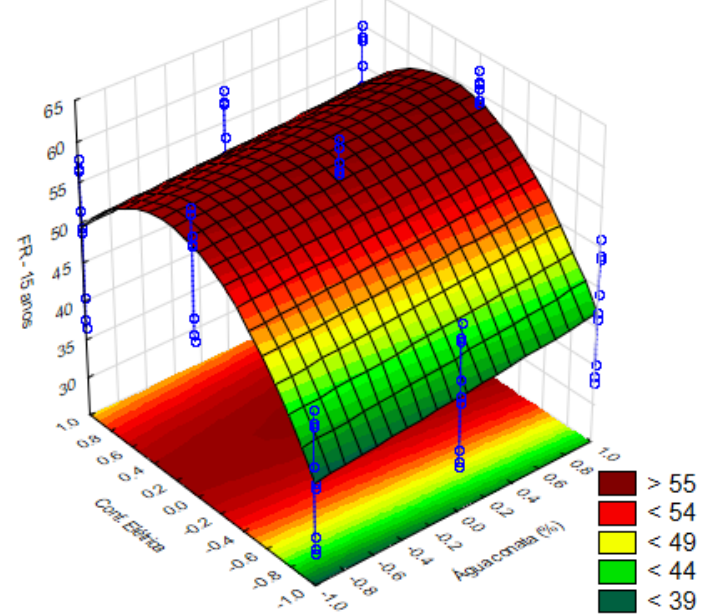

(a)

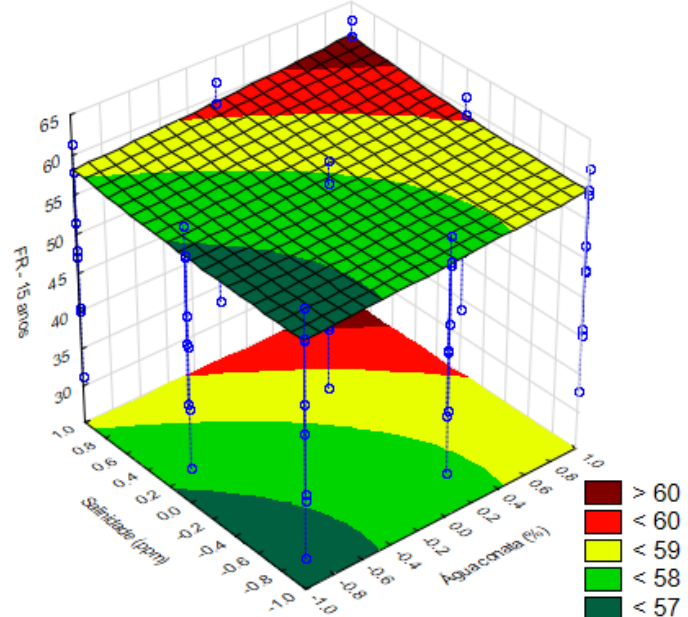

(b)

Figura 6. Superfícies de resposta de interação entre: (a) configuração elétrica de excitação dos eletrodos e saturação de água conata no reservatório e (b) teor de salinidade da água do reservatório e saturação de água conata. 
Portanto, com base na análise das superfícies de resposta, verifica-se que o fator de recuperação é maximizado pelas seguintes condições: maior saturação de água conata no reservatório $(40 \%)$, com maior teor de sal $(250.000 \mathrm{ppm})$, sendo o reservatório aquecido pelos eletrodos na configuração trifásica, excitados com maior nível de tensão (110 volts).

\section{CONCLUSÕES}

Esse trabalho tem como objetivo o estudo da aplicação do aquecimento resistivo em um reservatório de óleo pesado, inclusive a avaliação da influência de dois parâmetros de incerteza do reservatório (saturação de água conata e salinidade da água) e dois parâmetros operacionais (nível de tensão e configuração elétrica), na recuperação do óleo existente no reservatório. Embora tenham sido avaliados os intervalos de 5, 10 e 15 anos, verificou-se que os resultados eram convergentes, razão pela qual foram reproduzidos apenas os dados de 15 anos de projeto.

A avaliação da influência dos parâmetros de incerteza do reservatório levou à conclusão que a maior saturação de água conata, combinada com maior teor de salinidade dessa água, implica em maior fator de recuperação, mesmo que não impliquem necessariamente em maior volume de óleo recuperado, uma vez que maiores saturações de água significam menos óleo no volume poroso

A avaliação dos parâmetros operacionais levou à conclusão que a configuração trifásica de excitação de eletrodos, combinada com o maior nível de tensão,resulta em maior fator de recuperação, uma vez que faz fluir correntes elétricas maiores na água do meio poroso, que implicam em maior calor gerado pelo efeito Joule.

Entretanto, como essas configurações otimizadas implicam em circulação de corrente elétrica de maior intensidade, ocorre maior demanda de potência elétrica, consequentemente maior custo. Portanto, é importante que haja uma avaliação econômica dessas configurações, para verificar a implicação desses custos na viabilidade de algumas dessas configurações testadas.

A vantagem da abordagem apresentada está no fato dessa tecnologia poder ser vista como alternativa para os casos em que seja necessário reduzir a viscosidade do óleo no reservatório, mediante o aumento da temperatura, mas existam limitações à aplicação dos métodos térmicos que façam uso de vapor, principalmente no tocando ao consumo de água, que tem se tornado um recurso natural cada vez mais escasso.

Embora esse estudo faça uma indicação do potencial teórico da aplicação do aquecimento elétrico resistivo como método de recuperação, ele apresenta como desvantagem o fato do simulador numérico ignorar as limitações de ordem tecnológica, que têm sido verificadas nas tentativas de implementação prática. Dentre essas limitações, podem ser listadas a dificuldade de construção de grandes geradores de energia, para suprir as altas demandas de potência; a taxa de corrosão dos eletrodos, que aumenta em função do incremento das temperaturas e da operação dos eletrodos em meios agressivos; bem como as paradas do sistema para substituição desses eletrodos.

\section{AGRADECIMENTOS}

Os autores agradecem ao Programa de Pós-Graduação em Ciência e Engenharia de Petróleo (PPGCEP) e ao PRH-14, pelo apoio que têm dado ao desenvolvimento das pesquisas. Também 
agradecem ao Computer ModellingGroupLtd. (CMG), pela disponibilização de licenças do pacote de programas da $C M G$ Suite para a realização das simulações numéricas.O Programa Petrobras de Formação de Recursos Humanos forneceu apoio financeiro para o estudo.

\section{REFERÊNCIAS}

BAYLOR, B. A.; MAGGARD, J. B.; WATTENBARGER, R. A. Improved calculation of oil production response to electrical resistance heating (ERH). In: SPE ANNUAL TECHNICAL CONFERENCE AND EXHIBITION, 1990, New Orleans. Disponívelem: <https://Www.onepetro.org/conference-paper/SPE-20482-MS>. Acessoem: 25 Março 2014.

CARRIZALES, M. A. Recovery of stranded heavy oil by electromagnetic heating. 2010. 190f. Dissertation (Ph. D.) - Faculty of the Graduate School, University of Texas at Austin.

CURTIS, C.; KOPPER, R.; DECOSTER, E.; GUZMÁN-GARCIA, A.; HUGGINS, C.; KNAUER, L.; MINNER, M.; KUPSCH, N.; LINEARES, L. M.; ROUGH, H.; WAITE, M. Heavy-oil reservoirs. In: SCHLUMBERGER. Oilfield review, v.14, n.2, p.30-51, 2002.

KOVSCEK, A. R. Emerging challenges and potential futures for thermally enhanced oil recovery. JournalofPetroleum Science and Engineering, v.98-99, p.130-143, 2012.

PERASER, V.; PATIL, S. L.; KHATANIAR, S.; DANDEKAR, A. Y.; SONWALKAR, V. S. Evaluation of electromagnetic heating for heavy oil recovery from Alaskan Reservoirs. In: SPE WESTERN REGIONAL MEETING, 2012, Bakersfield. Disponívelem: <http://www.onepetro.org/mslib/servlet/onepetropreview?id=SPE-154123-MS>. Acesso em: 12 Novembro 2013.

OLIVEIRA, H. J. M. Estudo paramétrico da recuperação de petróleo pesado por aquecimento eletromagnético resistivo. 2009. 127f. Dissertação (Mestrado em Ciência e Engenharia de Petróleo), Programa de Pós-Graduação em Ciência e Engenharia de Petróleo, Universidade Federal do Rio Grande do Norte, Natal.

PIZARRO, J. O. S.; TREVISAN, O. V. Electrical heating of oil reservoirs: numerical simulation and field test results. JournalofPetroleum Technology, v.42, n.10, p.1320-1326, 1990.

SANTOS, M. D. Um modelo matemático baseado em wavelets para análise do método térmico de recuperação de óleo pesado aplicando irradiação eletromagnética. 2010. 82f. Tese (Doutorado em Ciência e Engenharia de Petróleo), Programa de Pós-Graduação em Ciência e Engenharia de Petróleo, Universidade Federal do Rio Grande do Norte, Natal.

SIERRA, R.; TRIPATHY, B.; BRIDGES, J. E.; FAROUQ ALI, S. M. Promising progress in field application of reservoir electrical heating methods. In: SPE INTERNATIONAL THERMAL OPERATIONS AND HEAVY OIL SYMPOSIUM, 2001, Margarita. Disponível em: <http://www.onepetro.org/mslib/app/Preview.do?paperNumber=00069709\&societyCode=SPE $>$. Acesso em: 22 Novembro 2013. 